\title{
ON EXTENSIONS OF VALUATIONS TO SIMPLE TRANSCENDENTAL EXTENSIONS
}

\author{
by SUDESH K. KHANDUJA and USHA GARG
}

(Received 1st October 1987)

\section{Introduction}

Let $v_{0}$ be a valuation of a field $K_{0}$ with residue field $k_{0}$ and value group $Z$, the group of rational integers. Let $K_{0}(x)$ be a simple transcendental extension of $K_{0}$. In 1936, Maclane [3] gave a method to determine all real valuations $V$ of $K_{0}(x)$ which are extensions of $v_{0}$. But his method does not seem to give an explicit construction of these valuations. In the present paper, assuming $K_{0}$ to be a complete field with respect to $v_{0}$, we explicitly determine all extensions of $v_{0}$ to $K_{0}(x)$ which have $Z$ as the value group and a simple transcendental extension of $k_{0}$ as the residue field. If $V$ is any extension of $v_{0}$ to $K_{0}(x)$ having $Z$ as the value group and a transcendental extension of $k_{0}$ as the residue field, then using the Ruled Residue theorem $[4,2,5]$, we give a method which explicitly determines $V$ on a subfield of $K_{0}(x)$ properly containing $K_{0}$.

In Section 1, we prove some results needed for the main results. These results, however, turn out to be of independent interest.

\section{Certain extensions of any real valuation to a simple transcendental extension}

In this section, $v_{0}$ is a real valuation of a field $K_{0}$ (not necessarily discrete or complete) with residue field $k_{0}$ and $K_{0}(x)$ is a simple transcendental extension of $K_{0}$. We shall denote by $V_{0}$ the valuation of $K_{0}(x)$ defined on $K_{0}[x]$ by

$$
V_{0}\left(\sum_{i=0}^{n} c_{i} x^{i}\right)=\min _{i} v_{0}\left(c_{i}\right)
$$

Let $P(x)$ be a monic polynomial with coefficients in the valuation ring $\mathrm{o}$ of $v_{0}$ such that the corresponding polynomial $\bar{P}(x)$ with coefficients in the residue field $k_{0}$ of $v_{0}$ is irreducible over $k_{0}$. Let $\theta$ be any positive real number. By successive division by powers of $P(x)$, any non-zero polynomial $f(x)$ in $\mathrm{o}[x]$ can be uniquely represented as

$$
f(x)=\sum_{i=0}^{m} f_{i}(x) P(x)^{i}
$$


where the polynomial $f_{i}(x)$ in $\mathrm{o}[x]$ is either zero or has degree less than that of $P(x)$. (The above representation of $f(x)$ will be referred to as the canonical representation of $f(x))$. We define $V_{P(x)}$ on $\mathfrak{o}[x]$ by

$$
V_{P(x)}(f(x))=\min _{i}\left(V_{0}\left(f_{i}(x)\right)+i \theta\right) .
$$

We shall soon prove that $V_{P(x)}$ is a valuation of $\mathrm{o}[x]$. Its unique extension to $K_{0}(x)$ will also be denoted by $V_{P(x)}$.

Lemma 1. If a non-zero polynomial $F(x)$ in $\mathrm{o}[x]$ is written, by the division algorithm, as

$$
F(x)=P(x) q(x)+r(x)
$$

then

$$
V_{0}(r(x)) \geqq V_{0}(F(x))
$$

and consequently

$$
V_{0}(q(x))=V_{0}(P(x) q(x)) \geqq V_{0}(F(x)) .
$$

Proof. This follows at once if we write $F(x)=\alpha F_{1}(x)$ with $\alpha$ in $\mathfrak{o}$ such that $V_{0}\left(F_{1}(x)\right)=0$ and then write by the division algorithm $F_{1}(x)$ as $P(x) q_{1}(x)+r_{1}(x)$.

The following remark follows immediately from above.

Remark 1. With notation as in Lemma $1, V_{0}(r(x))>V_{0}(F(x))$ if and only if $\bar{P}(x)$ divides $\bar{F}_{1}(x)$ over $k_{0}$, where $F_{1}(x)$ is any constant multiple of $F(x)$ with $V_{0}\left(F_{1}(x)\right)=0$.

Lemma 2. If $a(x)$ and $b(x)$ are two non-zero polynomials in $\mathfrak{o}[x]$, each of degree less than the degree of $P(x)$, then

$$
V_{P(x)}(a(x) b(x))=V_{P(x)}(a(x))+V_{P(x)}(b(x)) .
$$

Proof. Let $\alpha, \beta$ be elements of $\mathfrak{v}$ such that

$$
a(x)=\alpha a_{1}(x), b(x)=\beta b_{1}(x), V_{0}\left(a_{1}(x)\right)=V_{0}\left(b_{1}(x)\right)=0 .
$$

On dividing $a_{1}(x) b_{1}(x)$ by $P(x)$, we can write

$$
a_{1}(x) b_{1}(x)=P(x) q_{1}(x)+r_{1}(x) ;
$$

where either $r_{1}(x)=0$ or $\operatorname{deg} r_{1}(x)<\operatorname{deg} P(x)$. Since $\operatorname{deg}\left(a_{1}(x) b_{1}(x)\right)<\operatorname{deg} P(x)^{2}$, therefore $\operatorname{deg} q_{1}(x)<\operatorname{deg} P(x)$. Also $V_{0}\left(r_{1}(x)\right)=0$, i.e. the polynomial $\bar{r}_{1}(x) \neq 0$ in $k_{0}[x]$; for other- 
wise by (1.1) $\bar{P}(x)$ will divide $\bar{a}_{1}(x) b_{1}(x)$ and being irreducible must divide at least one of $\bar{a}_{1}(x)$ or $\bar{b}_{1}(x)$, which is impossible in view of the degrees of $\bar{a}_{1}(x)$ and $\bar{b}_{1}(x)$. On multiplying (1.1) by $\alpha \beta$, we have

$$
a(x) b(x)=P(x) \alpha \beta q_{1}(x)+\alpha \beta r_{1}(x) .
$$

Now by definition of $V_{P(x)}$, we have

$$
\begin{aligned}
& V_{P(x)}(a(x) b(x))=\min \left\{V_{0}\left(\alpha \beta q_{1}(x)\right)+\theta, V_{0}\left(\alpha \beta r_{1}(x)\right)\right\} \\
& =\min \left\{v_{0}(\alpha \beta)+V_{0}\left(q_{1}(x)\right)+\theta, v_{0}(\alpha \beta)\right\} \\
& =v_{0}(\alpha \beta)=V_{P(x)}(a(x))+V_{P(x)}(b(x)),
\end{aligned}
$$

and the lemma is proved.

Theorem 1. $V_{P(x)}$ is a valuation on $\mathrm{o}[x]$.

Proof. Let $f(x)$ and $g(x)$ be non-zero polynomials over $\mathbf{o}$ with canonical representations

$$
\begin{aligned}
& f(x)=\sum_{i=0}^{m} f_{i}(x) P(x)^{i}, f_{m}(x) \neq 0 \\
& g(x)=\sum_{j=0}^{n} g_{j}(x) P(x)^{j}, g_{n}(x) \neq 0 .
\end{aligned}
$$

On adding (1.2) and (1.3) we obtain the canonical representation for $f(x)+g(x)$ and the triangle law, i.e.,

$$
V_{P(x)}(f+g) \geqq \min \left\{V_{P(x)}(f), V_{P(x)}(g)\right\}
$$

follows immediately. Also it is easy to prove using Lemma 2 and the triangle law that

$$
V_{P(x)}(f g) \geqq V_{P(x)}(f)+V_{P(x)}(g) .
$$

Now, it remains to prove that

$$
V_{P(x)}(f g) \leqq V_{P(x)}(f)+V_{P(x)}(g) .
$$

Let $t$ and $u$ be the smallest indices such that

$$
V_{P(x)}(f(x))=V_{0}\left(f_{t}(x)\right)+t \theta, . V_{P(x)}(g(x))=V_{0}\left(g_{u}(x)\right)+u \theta .
$$


Let $r_{i}(x)$ and $q_{i}(x)$ be the polynomials over $\mathfrak{o}$ determined by the division algorithm from the following equations.

$$
\begin{gathered}
f_{0}(x) g_{0}(x)=q_{0}(x) P(x)+r_{0}(x) \\
f_{0}(x) g_{1}(x)+f_{1}(x) g_{0}(x)+q_{0}(x)=q_{1}(x) P(x)+r_{1}(x) \\
\vdots \vdots \vdots \\
\vdots \\
f_{m}(x) g_{n}(x)+q_{m+n-1}(x)=q_{m+n}(x) P(x)+r_{m+n}(x) .
\end{gathered}
$$

Observe that the degree of each $q_{i}(x)$ and $r_{i}(x)$ is less than the degree of $P(x)$. Thus the representation of $f(x) g(x)$ as

$$
f(x) g(x)=\sum_{i=0}^{m+n} r_{i}(x) P(x)^{i}+q_{m+n}(x) P(x)^{m+n+1}
$$

is the canonical representation.

The inequality (1.4) follows at once if we prove that

$$
V_{0}\left(r_{t+u}(x)\right)=V_{0}\left(f_{t}(x)\right)+V_{0}\left(g_{u}(x)\right) .
$$

We first show that

$$
V_{0}\left(f_{i}(x) g_{j}(x)\right)>V_{0}\left(f_{t}(x) g_{u}(x)\right) \quad \text { if } i+j<t+u
$$

and

$$
V_{0}\left(f_{i}(x) g_{j}(x)\right)>V_{0}\left(f_{t}(x) g_{u}(x)\right) \quad \text { if } i+j=t+u, \quad i \neq t .
$$

Both (1.6) and (1.7) follow immediately from the following observation.

For $0 \leqq i \leqq m, V_{0}\left(f_{i}\right)+i \theta \geqq V_{0}\left(f_{t}\right)+t \theta$ with strict inequality if $i<t$; and for $0 \leqq j \leqq n$, $V_{0}\left(g_{j}\right)+j \theta \geqq V_{0}\left(g_{u}\right)+u \theta$ with strict inequality if $j<u$.

Define a polynomial $F(x)$ over $\mathrm{o}$ by

$$
F(x)=\sum_{i+j=t+u} f_{i}(x) g_{j}(x)+q_{t+u-1}(x) .
$$

Recall that $q_{t+u}(x)$ and $r_{t+u}(x)$ are respectively the quotient and remainder when $F(x)$ is divided by $P(x)$ i.e.

$$
F(x)=q_{t+u}(x) P(x)+r_{t+u}(x) .
$$

In view of (1.6) and Lemma 1 , it is clear that

$$
V_{0}\left(q_{t+u-1}\right)>V_{0}\left(f_{t} g_{u}\right)
$$

In view of (1.7), we have 


$$
V_{0}\left(\sum_{i+j=t+u} f_{i} g_{j}\right)=V_{0}\left(f_{t} g_{u}\right)
$$

consequently

$$
V_{0}\left(f_{t} g_{u}\right)=V_{0}(F(x))
$$

Let $\alpha$ and $\beta$ be elements of $o$ such that $f_{t}(x)=\alpha F_{t}(x), g_{u}(x)=\beta G_{u}(x)$ with $V_{0}\left(f_{t}(x)\right)=v_{0}(\alpha)$ and $V_{0}\left(g_{u}(x)\right)=v_{0}(\beta)$.

Let $F_{1}(x)$ be the polynomial over $\mathrm{o}$ defined by $F(x)=\alpha \beta F_{1}(x)$. In view of (1.7) and $(1.8)$ it is clear that

$$
\bar{F}_{1}(x)=\bar{F}_{t}(x) \bar{G}_{u}(x)
$$

Since both $\bar{F}_{t}(x)$ and $\bar{G}_{u}(x)$ are of degree less than that of $\bar{P}(x)$, therefore $\bar{P}(x)$ does not divide $\bar{F}_{1}(x)$. It now follows from equation (1.9), Lemma 1 and the remark following the lemma that

$$
V_{0}\left(f_{t} g_{u}\right)=V_{0}(F)=V_{0}\left(r_{t+u}\right)
$$

which proves (1.5) and hence completes the proof of the fact that $V_{P(x)}$ is a valuation of $\mathrm{o}[x]$.

Notation. If $\alpha$ is an element of the valuation ring of a valuation $V$ of a field $K$, then $\bar{\alpha}$ will denote its image in the residue field of $V$.

The following theorem determines the residue fields of the valuations $V_{0}$ and $V_{P(x)}$. The residue field of $V_{0}$ is wellknown $[1, \S 10.2$, Prop. 2]. For the sake of completeness we determine it here also.

Theorem 2. With $v_{0}, k_{0}, V_{0}, \theta, V_{P(x)}$ as before and with $G_{0}$ as the value group of $v_{0}$, we have:

(i) The residue field of the valuation $V_{0}$ is $k_{0}(\bar{x})$ with $\bar{x}$ (the image of $x$ in the residue field of $V_{0}$ ) transcendental over $k_{0}$.

(ii) If $\theta$ is free modulo $G_{0}$, then the residue field of $V_{P(x)}$ is $k_{0}(\bar{x})$ with $\bar{x}$ (the image of $x$ in the residue field of $\left.V_{P(x)}\right)$ algebraic over $k_{0}$.

(iii) If $\theta$ is torsion modulo $G_{0}$, with $s$ as the smallest positive integer such that $s \theta\left(=v_{0}(\alpha)\right)$ is in $G_{0}$, then the residue field of $V_{P(x)}$ is $k_{0}[\bar{x}](t)$ where $t=$ the residue class of $P(x)^{s} / \alpha$ (in the residue field of $V_{P(x)}$ ), is transcendental over $k_{0}[\bar{x}]$ and $\bar{x}$ is algebraic over $k_{0}$.

Proof. In all the three cases, we denote by $\Delta$ the residue field of the valuation under 
consideration and by $\bar{\xi}=(f(x) / g(x))^{-}$an arbitrary non-zero element of $\Delta$, with $f(x)$ and $g(x)$ in $\mathrm{o}[x]$.

(i) It is easy to verify that the image $\bar{x}$ of $x$ in the residue field of $V_{0}$ is transcendental over $k_{0}$ in this case. Let $\beta$ be an element of $\mathrm{o}$ such that

$$
V_{0}(f(x))=V_{0}(g(x))=v_{0}(\beta) .
$$

Then

$$
V_{0}(f(x) / \beta)=0, V_{0}(g(x) / \beta)=0 .
$$

So $\bar{\xi}=\bar{\xi}_{1} \bar{\xi}_{2}^{-1}$ where $\bar{\xi}_{1}=(f(x) / \beta)^{-}$and $\bar{\xi}_{2}=(g(x) / \beta)^{-}$are in $k_{0}[\bar{x}]$. This proves that $\Delta=k_{0}(\bar{x})$.

(ii) Let

$$
\begin{aligned}
& f(x)=\sum_{i} f_{i}(x) P(x)^{i} \\
& g(x)=\sum_{j} g_{j}(x) P(x)^{j}
\end{aligned}
$$

be the canonical expression for $f(x)$ and $g(x)$ respectively. For non-zero polynomials $f_{i}(x)$ and $g_{j}(x)$, define polynomials $F_{i}(x)$ and $G_{j}(x)$ with coefficients in $o$ by

$$
f_{i}(x)=\beta_{i} F_{i}(x), g_{j}(x)=\gamma_{j} G_{j}(x)
$$

where $v_{0}\left(\beta_{i}\right)=V_{0}\left(f_{i}(x)\right)$ and $v_{0}\left(\gamma_{j}\right)=V_{o}\left(g_{j}(x)\right)$. Thus

$$
\begin{aligned}
& f(x)=\sum_{i} \beta_{i} F_{i}(x) P(x)^{i}, \\
& g(x)=\sum_{j} \gamma_{j} G_{j}(x) P(x)^{j} .
\end{aligned}
$$

Since $V_{P(x)}(f(x))=V_{P(x)}(g(x))$, i.e., $\min _{i}\left(v_{0}\left(\beta_{i}\right)+i \theta\right)=\min _{j}\left(v_{0}\left(\gamma_{j}\right)+j \theta\right)$, therefore there exist subscripts $h$ and $k$ such that

$$
v_{0}\left(\beta_{h}\right)+h \theta=v_{0}\left(\gamma_{k}\right)+k \theta
$$

In this case, $\theta$ being free modulo $G_{0}$, the above equality is possible only if $h=k$ and $v_{0}\left(\beta_{h}\right)=v_{0}\left(\gamma_{h}\right)$, also $v_{0}\left(\beta_{i}\right)+i \theta>v_{0}\left(\beta_{h}\right)+h \theta$ if $i \neq h$ and $v_{0}\left(\gamma_{j}\right)+j \theta>v_{0}\left(\beta_{h}\right)+h \theta$ if $j \neq h$. So if we write $\xi_{1}=f(x) / \beta_{h} P(x)^{h}$ and $\xi_{2}=g(x) / \beta_{h} P(x)^{h}$, we have $\xi=\xi_{1} / \xi_{2}=$ $\bar{F}_{h}(\bar{x})\left(\left(\gamma_{h} / \beta_{h}\right)^{-} \bar{G}_{h}(\bar{x})\right)^{-1}$ is in $k_{0}(\bar{x})$; here $\bar{G}_{h}(\bar{x}) \neq 0$, because the degree of the polynomial $\bar{G}_{h}(y)$ is less than the degree of $\bar{P}(y)$ which is the minimal polynomial of $\bar{x}$ over $k_{0}$. This proves that $\Delta=k_{0}(\bar{x})$ which is an algebraic extension of $k_{0}$.

(iii) Let $s$ be the smallest positive integer such that $s \theta \in G_{0}$, (say) $s \theta=v_{0}(\alpha)$ with $\alpha$ in $\mathbf{0}$. 
We first prove that the residue class $\left(P(x)^{s} / \alpha\right)^{-}=t$ (say) is transcendental over $k_{0}$. Suppose $t$ is algebraic over $k_{0}$. Let $y^{m}+\bar{a}_{1} y^{m-1}+\cdots+\bar{a}_{m}$ be a polynomial over $k_{0}$ satisfied by $t$. Therefore

$$
V_{P(x)}\left(\left(P(x)^{s} / \alpha\right)^{m}+a_{1}\left(P(x)^{s} / \alpha\right)^{m-1}+\cdots+a_{m}\right)>0
$$

i.e., if we write

$$
F(x)=P(x)^{s m}+a_{1} \alpha P(x)^{s(m-1)}+\cdots+a_{m} \alpha^{m}
$$

then

$$
V_{P(x)}(F(x))>v_{0}\left(\alpha^{m}\right)=m s \theta,
$$

which is impossible because (1.12) is a canonical expression for $F(x)$ and by definition of $V_{P(x)}$, we must have $V_{P(x)}(F(x)) \leqq s m \theta$. This contradiction proves that $t$ is transcendental over $k_{0}$; in fact $t$ is transcendental over $k_{0}(\bar{x})$ because $\bar{x}$, satisfying the polynomial $\bar{P}(y)$, is algebraic over $k_{0}$.

Let expressions for $f(x)$ and $g(x)$ be as in (1.10) and (1.11). Let $\beta$ be an element of $\mathfrak{v}$ and $h$ an integer such that

$$
v_{0}(\beta)+h \theta=\min _{i}\left(v_{0}\left(\beta_{\mathrm{i}}\right)+i \theta\right)=\min _{j}\left(v_{0}\left(\gamma_{\mathrm{j}}\right)+j \theta\right)
$$

Write $\xi_{1}=f(x) / \beta P(x)^{h}, \xi_{2}=g(x) / \beta P(x)^{h}$. Then

$$
\bar{\xi}_{1}=\sum_{i}^{\prime} \bar{F}_{i}(\bar{x})\left(\beta_{i} P(x)^{i} / \beta P(x)^{h}\right)^{-}
$$

where the sum $\sum^{\prime}$ is carried over all those $i$ for which $v_{0}\left(\beta_{i}\right)+i \theta=v_{0}(\beta)+h \theta$ (the rest of the terms are zero in the residue field). For each $i$ in $\sum^{\prime}, v_{0}\left(\beta_{i}\right)+i \theta=v_{0}(\beta)+h \theta$, i.e., $(i-h) \theta=v_{0}(\beta)-v_{0}\left(\beta_{i}\right)$. So $(i-h)$ is an integral multiple of $s$, say $(i-h)=m_{i}$ s. Therefore the residue class of $\beta_{i} P(x)^{i} / \beta P(x)^{h}=\left(P(x)^{s} / \alpha\right)^{m_{i}} \cdot\left(\beta_{i} \alpha^{m_{i}} / \beta\right)$ is an integral power of $t=\left(P(x)^{s} / \alpha\right)^{-}$multiplied by an element of $k_{0}$. Thus $\bar{\xi}_{1}$ is in the field $k_{0}[\bar{x}](t)$. Similarly $\bar{\xi}_{2}$ and hence $\bar{\xi}$ are in the same field. This proves (iii).

Remark 2. As in [1, $\S 10.2$, Prop. 2], it is easy to prove that if $V$ is a real valuation of $K_{0}(x)$ extending the valuation $v_{0}$ of $K_{0}$ with $V(x)=0$ and if $\bar{x}$ is transcendental over $k_{0}$ then $V=V_{0}$.

\section{Construction of extensions of $v_{0}$ with residue field $k_{0}(t)$}

In what follows, $K_{0}$ is a complete valuation field with respect to a valuation $v_{0}$ having the value group $Z$, the valuation ring $\mathfrak{o}$ and the residue field $k_{0}$. As before $x$ is an indeterminate, We shall consider only those extensions $V$ of $v_{0}$ to $K_{0}(x)$ for which $V(x) \geqq 0$. 
Theorem 3. Let $V$ be an extension of $v_{0}$ to $K_{0}(x)$ with value group $Z$ and residue field $\Delta$ such that $\Delta \cap\left(\right.$ the algebraic closure of $\left.k_{0}\right)=k_{0}(\bar{x})$, then $V=V_{P(x)}$ for some monic polynomial $P(x)$ over $\mathfrak{o}$ where $\bar{P}(y)$ is the minimal polynomial of $\bar{x}$ over $k_{0}$.

Proof. Let $\pi$ be a uniformizer of $v_{0}$ in $K_{0}$ and $\phi(y)$ be the minimal polynomial of $\bar{x}$ over $k_{0}$ of degree $n$. We claim that there exists a monic polynomial $P(x)$ over $\mathfrak{o}$ with $\bar{P}(x)=\phi(x)$ such that the residue class $\left(P(x) / \pi^{n}\right)^{-}$(in the residue field of $V$ ) is transcendental over $k_{0}, r$ being given by $V(P(x))=r$. Let $P_{1}(x)$ be any monic polynomial with coefficients in $\mathrm{o}$ such that $\bar{P}_{1}(x)=\phi(x)$. Since $\bar{P}_{1}(\bar{x})=\phi(\bar{x})=\overline{0}$, therefore $s_{1}=$ $V\left(P_{1}(x)\right)>0$. If $\left(P_{1}(x) / \pi^{s_{1}}\right)^{-}$is transcendental over $k_{0}$ then our claim is proved. If it is algebraic over $k_{0}$ then by hypothesis there exists a polynomial $f_{1}(x)$ in $\mathrm{o}[x]$ of degree $\leqq n-1$ such that

$$
\left(P_{1}(x) / \pi^{s_{1}}\right)^{-}=\bar{f}_{1}(\bar{x})
$$

So

$$
V\left(P_{1}(x)-\pi^{s_{1}} f_{1}(x)\right)>s_{1} .
$$

Write $P_{2}(x)=P_{1}(x)-\pi^{s_{1}} f_{1}(x)$ and define an integer $s_{2}>s_{1}$ by $V\left(P_{2}(x)\right)=s_{2}$. If $\left(P_{2}(x) / \pi^{s_{2}}\right)^{-}$is transcendental over $k_{0}$, we stop here otherwise we continue the process. We show that the process cannot continue indefinitely. Suppose it does. So we obtain a sequence of polynomial $f_{i}(x)$ in $\mathfrak{o}[x]$ each of degree $\leqq n-1$ and a strictly increasing sequence of positive integers $s_{1}<s_{2}<\cdots$ such that

$$
V\left(P_{1}(x)-\sum_{j=1}^{t} f_{j}(x) \pi^{s_{j}}\right)=s_{t+1}
$$

Since $K_{0}$ is complete and since each $f_{j}(x)$ is of degree $\leqq n-1$, therefore $\sum_{j=1}^{\infty} f_{j}(x) \pi^{s_{j}}$ is a polynomial over $\mathfrak{o}$ of degree $\leqq n-1$, which we shall denote by $F(x)$. By choice $V\left(P_{1}(x)-F(x)\right)>s_{t}$ for all $t$, so $P_{1}(x)-F(x)$ must be the zero polynomial. Which is impossible because $P_{1}(x)-F(x)$ is a monic polynomial of degree $n$ over $\mathfrak{o}$. This contradiction proves the claim.

Let $P(x)$ be a monic polynomial over $\mathfrak{o}$ such that $\bar{P}(x)=\phi(x), \quad V(P(x))=r$ and $\left(P(x) / \pi^{r}\right)^{-}$is transcendental over $k_{0}$ and hence over $k_{0}(\bar{x})$. We now prove that the valuation $V$ is nothing but $V_{P(x)}$ with $\theta=V_{P(x)}(P(x))=r$. Since the minimal polynomial satisfied by $\bar{x}$ over $k_{0}$ has degree $n$, therefore for any polynomial $a(x)$ over $o$ of degree $\leqq n-1, V(a(x))=V_{0}(a(x))$ holds. Let $f(x)$ be any non-zero element of $\mathrm{o}[x]$ and let

$$
f(x)=\sum_{i=1}^{m} f_{i}(x) P(x)^{i}
$$

be the canonical representation of $f(x)$. If $f_{i}(x) \neq 0$ then $\operatorname{deg} f_{i}(x) \leqq n-1$. So

$$
V\left(f_{i}(x)\right)=V_{0}\left(f_{i}(x)\right)=a_{i}, \quad \text { (say) }
$$


By definition of $V_{P(x)}$, we have

$$
V_{P(x)}(f(x))=\min _{i}\left(a_{i}+i r\right)
$$

where the minimum is carried over those $i$ for which $f_{i}(x) \neq 0$. We shall prove that

$$
V(f(x))=\min _{i}\left(a_{i}+i r\right)=V_{\mathbf{P}(x)}(f(x)) .
$$

Since $V\left(f_{i}(x) P(x)^{i}\right)=a_{i}+i r$, it follows from (2.1) that

$$
V(f(x)) \geqq \min _{i}\left(a_{i}+i r\right) .
$$

We now prove (2.2). Let $h$ (be the smallest subscript such that $\min _{i}\left(a_{i}+i r\right)=a_{h}+h r$. For a non-zero polynomial $f_{i}(x)$ define $F_{i}(x)$ in $\mathrm{o}[x]$ by $f_{i}(x)=\pi^{\alpha_{i}} F_{i}(x)$, so that $V_{0}\left(F_{i}(x)\right)=$ $V\left(F_{i}(x)\right)=0$. Suppose strict inequality holds in (2.3). Then there exist positive integers $h=h_{0}<h_{1}<\cdots<h_{l} \leqq m$, such that

$$
V\left(\sum_{i=0}^{l} \pi^{a_{h_{i}}} F_{h_{i}}(x) P(x)^{h_{i}}\right)>a_{h}+h r
$$

and

$$
V\left(\pi^{a_{h_{1}}} F_{h_{i}}(x) P(x)^{h_{i}}\right)=a_{h}+h r
$$

for $0 \leqq i \leqq l$. It follows from (2.5) that

$$
\left(a_{h}-a_{h_{i}}\right)=\left(h_{i}-h_{0}\right) r=n_{i} r, \quad \text { (say) }
$$

for $1 \leqq i \leqq l$. Observe that $n_{1}<n_{2}{ }^{\prime}<\cdots<n_{l}$. It follows at once from (2.4) and (2.6) that

$$
V\left(F_{h_{0}}(x)+\sum_{i=1}^{l}\left(P(x) / \pi^{r}\right)^{n_{i}} F_{h_{i}}(x)\right)>0
$$

which shows that $\left(P(x) / \pi^{r}\right)^{-}$is algebraic over $k_{0}[\bar{x}]$. This contradiction proves that equality holds in (2.3) and hence $V=V_{P(x)}$.

Remark 3. If $V$ is as in the above theorem then we have shown in Theorem 2, part (iii) of Section 1 that the residuce field of $V$ is a simple transcendental extension of $k_{0}(\bar{x})$.

Theorem 4. Let $V$ be an extension of $v_{0}$ to $K_{0}(\bar{x})$ with value group $Z$ and residue field a simple transcendental extension of $k_{0}$ then either $V=V_{0}$ or $V=V_{P(x)}$ for some monic linear polynomial $P(x)$ over 0 . 
Proof. If $\bar{x}$ is transcendental over $k_{0}$ then by the remark in the end of Section 1 , $V=V_{0}$. Suppose now that $\bar{x}$ is algebraic over $k_{0}$, therefore $\bar{x}$ is in $k_{0}$. So the minimal polynomial of $\bar{x}$ over $k_{0}$ is a linear polynomial. The desired assertion now follows immediately from Theorem 3 .

\section{Method of construction of valuations with residue field transcendental over $\boldsymbol{k}_{\mathbf{0}}$}

Notation and assumptions are as in the previous section. Now we assume that $k_{0}$ is a perfect field. Let $V$ be an extension of $v_{0}$ to $K_{0}(x)$ with value group $Z$ and residue field $\Delta$ transcendental over $k_{0}$. By the Ruled Residue theorem [5], there exists a finite extension $k_{1}$ of $k_{0}$ such that $\Delta=k_{1}(t)$ with $t$ transcendental over $k_{0}$. If $k_{1}=k_{0}$, then by Theorem 4, either $V=V_{0}$ or $V=V_{P_{(x)}}$ where $P(x)$ is a linear polynomial over $\mathrm{o}$. Suppose now that $k_{1}$ is a proper extension of $k_{0}$. Since $k_{0}$ is perfect, therefore there exists $y$ in $K_{0}(x)$ such that $V(y)=0$ and $k_{1}=k_{0}(\bar{y})$. Then $y$ does not belong to $K_{0}$, so $K_{0}(x)$ is a finite extension of $K_{0}(y)$. Let $V_{1}$ denote the restriction of $V$ to $K_{0}(y)$. The hypotheses of Theorem 3 are clearly satisfied for the valuation $V_{1}$ of $K_{0}(y)$, so by this theorem $V_{1}=V_{P(y)}$ for some monic polynomial $P(y)$ with coefficients in 0 . Thus the valuation $V$ is completely determined on $K_{0}(y)$.

\section{REFERENCES}

1. N. Bourbaki, Commutative Algebra, Chapter 6, Valuations (Hermann, Publishers in arts and science, 1972).

2. W. J. Heinzer, Valuation rings and simple transcendental field extensions, J. Pure Appl. Algebra 26 (1982), 189-190.

3. S. Maclane, A construction for absolute values in polynomial rings, Trans. Amer. Math. Soc. 40 (1936), 363-395.

4. M. NAGATA, A theorem on valuation rings and its applications, Nagoya Math. J. 29 (1967), 85-91.

5. Ј. Онм, The ruled residue theorem for simple transcendental extensions of valued fields, Proc. Amer. Math. Soc. 89 (1983), 16-18.

Department of Mathematics

Panjab University

Chandigarh-160014

INDIA 